\title{
Formation of interpersonal, group and organizational communications skills of future economists
}

\author{
Olga Kruchina ${ }^{1, *}$, Viktoria Skorobogatova ${ }^{1}$, and Tatyana Smirnova ${ }^{2,}$ \\ ${ }^{1}$ Kerch State Maritime Technological University, 82 Ordzhonikidze st., Kerch, 298309, Russia \\ ${ }^{2}$ Sevastopol State University, 33 University st., Sevastopol, 99000, Russia
}

\begin{abstract}
The purpose of the research is an identification, theoretical justification and experimental verification of pedagogical technologies that contribute to the efficiency of future specialist's professional and communicative skills formation as well as a role justification of sociopsychological qualities in the effectiveness of the process of communicative interaction in order to determine the main indicators of readiness for communicative interpersonal and group interaction. The object of our study is interpersonal, group and organizational communications skills and the subject of this one is characteristic of communicative interaction indicators. The following paper draws on material of different psychological researches and educational normative documents. The analysis of Federal State Educational Standard of Higher Education in the directions of training 38.03.01 Economy (a bachelor degree) was provided which showed that interpersonal communication skills possession is one of necessary competence nowadays. The authors emphasized that it is important to take into consideration sociopsychological qualities of future economists as a basics of their professionalism. Scientific and methodological recommendations and conclusions are obtained based on a theoretical analysis of the sociopsychological qualities of economists that provide the efficiency of the formation process of future economists' communicative interaction in the context of professional training.
\end{abstract}

\section{Introduction}

The economic market is developing so fast that a constant problem is the low level of staff training. This problem shows the need to develop future specialists' skills which provide competitiveness in the modern labor market. Every specialist is required to communicate with and interact with a wide range of people, from suppliers and customers through to immediate colleagues, colleagues, the team and manager. The lack of the necessary number of specialists with the appropriate level of qualification is the main constraint on the development of a modern and promising sector of the economy. Customer-facing roles,

* Corresponding author: scorpion19.11@yandex.ru 
such as sales and customer relations management, are likely to specify good interpersonal skills as a pre-requisite.

The regulatory requirements for professional and personal training of specialists are growing accordingly with the rapid development of the economy, increasing the overall level of communication competence. It is known that students with the most developed communication skills have a greater chance of getting an invitation to a prestigious job before the end of their training period. In the conditions of increasing competition in the market, this trend is most clearly expressed in the extreme demand for "communicative advanced" graduates of the economic profile. This means that the educational services market will benefit those universities which prepare specialist with communicative skills. They are open to interaction and quickly adapt to any organizational environment. These characteristics are considered as important criteria for their professionalism, as well as indicators of the quality of training of future economists in higher education institutions.

\subsection{Relevance of the research}

The relevance of this study is determined by the social demand of society for better training of economists in universities, since the economy is one of the main areas of future development.

Many scientists emphasize the importance of economists' possession of such qualities as the ability to abstract and understand the relationships between elements, flexibility of thinking, criticality, a propensity to plan, analyze and work systematically, and a willingness to replenish knowledge and retrain. The most important overarching personal skill is stated to be developing emotional intelligence as the ability to understand others emotions, and their effect on behaviour and attitudes.

\subsection{Study of the problem}

Daniel Goleman, the author of some books on emotional intelligence, identified key areas: the personal skills, or 'how we manage ourselves' motivation, self-regulation, are selfawareness; the social skill, or 'how we handle relationships with others', is empathy (understanding and feeling for others, and then being able to interact effectively with them). He considers that the first steps towards understanding and managing the emotions of others is to be able to understand and manage our own emotions, including understanding what motivates us.

Scientists A.R. Akhmadullin, V.V. Rubtsov, G.S. Tseitin, M. Bezpartochnyi, I.P. Shkuratova, D. Knut, T.S. Bochkareva, S. Sonnentag, and others, were engaged in questions about the role of socio-psychological qualities in the professional development of a specialist. They believe that a good specialist should be able to work with other people in a team and with customers.

Accelerating economic and social progress requires the development of innovative approaches to the educational process.

The quality of modern education has become an important subject of discussion of the pedagogical community. According to A.I. Subetto "the management of the future is possible in terms of development of human qualities, social intelligence and educational systems in society".

The higher school is intended to provide certain conditions which provide the possibility of "transformation of personal and social goals into an effective internal incentive for professional and creative development of the future specialist, the development of an active professional position and creative style of activity» $[1,3]$. 
A review of domestic and foreign sources and pedagogical practice shows that there is the following contradiction in modern professional education: between the need for specialists, which are ready to implement personal and socially significant professional communicative activities for solving professional tasks effectively, and insufficient development of methodical bases and substantial support of the process of formation of this readiness in terms of social-psychological qualities of the personnel.

\section{The aim of the research}

The purpose of this article is an identification, theoretical justification and experimental verification of pedagogical technologies that contribute to the efficiency of future specialist's professional and communicative skills formation as well as a role justification of socio-psychological qualities in the effectiveness of the process of communicative interaction in order to determine the main indicators of communicative interpersonal and group interaction. The object of this research is interpersonal, group and organizational communications skills. The subject of one is indicators of future specialists for communicative interpersonal and group interaction.

In accordance with the goal, the following tasks were set:

1) to determine the role of socio-psychological qualities in the effectiveness of the process of communicative interaction in the future professional activity;

2) to consider innovative technologies in order to improve the level of students' professional communication skills.

\section{Materials and methods}

To achieve the goal, we used a set of complementary theoretical research methods: analysis of philosophical, sociological, psychological, pedagogical, social, and economics literature on the problem under consideration; analysis of documentation on the organization of the educational process at the university for the theoretical justification of the essence, functions, and indicators of future specialists' communicative interaction skills, analysis, synthesis, and systematization of the main approaches to solving the research problem.

\section{Results and discussion}

The theoretical analysis of the personnel state of economy shows that there is a problem of acute personnel shortage and a low share of employment in this sphere. Many researchers have established an unsatisfactory professional and qualification level of specialists and a low attractiveness of one for young people.

Analysis of the Federal State Educational Standard of Higher Education in the specialty 38.03.01 Economy (bachelor's level) showed that among the necessary skills are the following:

- An ability to communicate orally and in writing in Russian and foreign languages to solve problems of interpersonal and intercultural interaction (General Competence -4);

- An ability to work in a team, tolerant of social, ethnic, religious and cultural differences (General Competence -5);

- ability to find organizational and managerial solutions in professional activities and willingness to take responsibility for them (General Professional Competence -4).

Organizational and management activities include the fillowing competences

- an ability to organize the activities of a small group created for the implementation of a specific economic project (Professional Competence-9); 
- an ability to use modern technical tools and information technologies for solving communication problems (Professional Competence -10);

- a ability to critically evaluate the proposed options for management decisions and develop and justify proposals for their improvement, taking into account the criteria of socio-economic efficiency, risks and possible socio-economic consequences (Professional Competence -11) [2,3].

Having analyzed the current state of higher education, we concluded that in the conditions of modern scientific and technological revolution there is a gap between the historical tradition of learning in higher education and the needs of society. In the content of the modernization of the professional education system, a transition to the use of modern methods and technologies of training aimed at improvement of communicative skills and motivation [4].

Professional standards and qualifications "Finance and Economics" determines that future economists must know the Basics of Psychology and Sociology of work and follow the rules of business communication ethics [1,4].

Interpersonal skills are considered to be ones required to effectively communicate, interact, and work with groups or individuals. Managers with good interpersonal skills are strong verbal and non-verbal communicators. These skills are important for success and can help to create good working relationships, manage conflict, solve problems, motivate the team, increase productivity, and increase engagement at work. Communicative skills can be defined as certain attributes or abilities that an executive should possess in order to fulfill specific tasks in an organization. They are managerial culture, intelligence, psychological competence, leadership skills, an ability to manage conflicts, tolerance, collegiality, optimism, extroversion, rhetorical abilities, sense of humor, ability to create a positive image-sociability, as willingness and need to communicate with other people, openness to other people [5].

It should be taken into consideration that the psychological aspect of communication significantly enriches the process of bilateral information exchange in interpersonal communication, and has a regulatory impact on the course of interaction.

The solution of these problems at the level of the organization is possible in a complex work, which is based on a thorough study of the specifics of labor activity, the role of professional and socio-psychological characteristics of a modern successful specialist in the process effectiveness of communicative interaction. «It is acmeology that solves a very difficult task: to find out features of a person on each phase of his/her life that he/she must reach "acme" in communication process [7]. The subject of it, therefore, is to ensure adequate socialization process, social adaptation and resocialization of the individual in certain periods of development.

Interpersonal relationships can be attributed to the selective activity of a person in the process of socialization. Personal characteristics of a person encourage other subjects to communicate.

We determine the following socio-psychological qualities of economists on the basics of various sources analysis:

- activity as an ability to act vigorously and make quick decisions;

- practicality of mind-ability to apply knowledge in any situation;

- communication skills as an ability to establish contact with people quickly;

- empathy as an ability to empathize, understand the mood of people, identify their attitudes and expectations;

- self-control, self-criticism, self-assessment of the actions;

- stress resistance as a self-hypnosis, the ability to manage your emotions.

At the same time, practice shows that a significant number of economists underestimate the personal factor in the effectiveness of their activities and professional development, and 
cannot use such personal qualities as the ability to take reasonable risks, resist stress, sociability, politeness, independence, and independence in solving managerial tasks. They have high anxiety which is expressed in poor adaptability, dissatisfaction with the achieved and high level of anxiety. Modern economists don't have a visual culture of communication, goodwill and respect for business partners. A specific analysis of practice shows that managers often violate the ethical norms of business interaction (noncompliance with such moral requirements of decency, modesty, justice, responsibility, honesty, etc.). Economists often do not realize that the external side of the economists' personality is not only a manifestation of internal culture, but also professionalism.

Good communicative skills are vital for any organization to succeed and achieve its objectives. An economist who fosters good communicative skills is able to propel the company's strategy or business purposes forward with fewer hurdles and objections from internal and external sources.

Leadership and communicative skills are often used interchangeably as they both include communication, decision-making, planning, time management problem-solving and delegation.

Social psychologist Robert L. Katz has set three areas of managerial skills and determination, for which level they are characterized: Technical Skills, Human Skills and Conceptual Skills $[1,7]$.

Social psychologist Robert L. Katz in his article "Skills of an Effective Administrator" in Harvard Business thought about the relationship of managerial skills (competences) and hierarchical management levels [5,7]. The result was the setting of the three areas of managerial skills and determination, for which level they are characterized:

"Technical skills - competencies important, particularly for lower management

Human skills - competencies needed for all levels of management

Conceptual skills - competencies with a substantial importance, particularly for top management" [1].

The human (the interpersonal skills) are ones that present the economists' ability to interact, relate or work effectively with people. These skills give possibility for the economists to make use of human potential in the company and motivate every person for better results. Possessing great communication skills is crucial for an economist. It can determine how well information is shared throughout a team, ensuring that the group behaves as a unified workforce. How well an economist communicates with the rest of his/her team also determines, how well the tasks and activities can be completed, and thus, how successful an organization will be [3].

The second task of this research is to consider innovative technologies in order to improve the level of students' professional interpersonal communication skills.

Innovative pedagogical technologies provide independence of experimental and research orientation of training. Role-playing games and communicative professional trainings are actively involved in the educational process. Synthesis of two techniques ("training" and "game") provide the solution of important tasks on communicative adaptation of future specialists, such as: phonemic speech; automation of speech normalization; methodological and practical orientation in the field of research, the development of stylistic "flair"; the creation of creative personality (creative) motivation; expanding the ideological base.

Communication-training games are innovative teaching technologies, aimed at modeling specific communicative situations and allowing students not only to practice their theoretical knowledge, but also learn to adapt to the designated context (not always positive), and to analyze speech and nonverbal behavior of game participants.

In recent years, professional organizations selects such specialists who not only have professional knowledge and skills and motivated to perform their duties, but also know and 
understand the principles of productive team work, approaches that contribute to the effectiveness of products, strategies and practices, and which are ready to establish harmonious relationships with colleagues in the process of interaction, have an "open" character in communication; the desire for awareness; a high level of claims and ambitious desire to assert the identity; the ability to establish business relationships; the ability to overcome a conflict situation; the ability to listen and convince.

Monitoring the development of appropriate communication skills can significantly improve the quality of professional training and, as a result, the internal efficiency of future specialists. In this case, the role of practical psychologists in the psychological support of professional activities of specialists is important. The main tasks of psychologists can be formulated as follows: conduct research to improve the quality of professional activity of these specialists in accordance with their psychophysiological capabilities and taking into account situations in accepted practice.

Currently, the Myers-Briggs psychological test is actively used to diagnose significant socio-psychological qualities of specialists, which is based on the theory of psychological types developed by K. Jung (Jung).

The Myers-Briggs diagnostic system has been widely used in business, in major western companies. The essence of the MBTI psychological testing system is that by measuring unique combinations of personal factors, it is possible to predict their propensity to a certain type of activity, the style of actions, the nature of decisions and other features that allow to feel comfortable and confident. 4 scales (descriptors), by which the personality is studied:

- orientation of consciousness (introversion-extroversion);

- orientation in a situation (common sense-intuition);

- basis of decision-making (logos-pathos);

- method of preparing solutions (rationality - irrationality).

There are also professional-oriented questionnaires that are specifically designed for selecting specialists, including questions and practical tasks aimed at assessing their ability to inter-organizational cooperation. In addition, many successful organizations identify professional and personal qualities during job interviews, as well as during the assigned probation period, which increases probability of exception of those specialists who do not have certain skills and increases the relevance of developing appropriate communication skills at the stage of general professional training.

Pedagogical diagnostics was carried out as a basis for the implementation of pedagogical monitoring, which is a prerequisite for the development of educational activities.

Pedagogical monitoring assumed tracking of activity parameters, as well as monitoring changes in the state of the researched object in specially created educational conditions. In general, monitoring was a specially designed, targeted, long-term and planned system of actions.

Monitoring provided the implementation of the following functions that reveal the characteristics of educational activity: study of the state of the educational system to obtain operational information about its specifics. In this way, the system can be adjusted in the direction of improvement.

- identification of changes in the educational system, as well as the factors that caused them.

- prevention of negative trends and risks in educational activities.

- providing the completeness of implementation and evaluating the effectiveness of methodological support for educational activities.

To study the level of formation of communicative skills of university students, we conducted an ascertaining experiment. Its purpose was to study the development indicators 
of each of the components. The main methods were chosen as a questionnaire, conversation, ranking, and observation. The study involved 100 students of the economics specialties of Kerch State Maritime Technological University. We used the methods "Value orientations "by M. Rokich,"Express diagnostics of social values of the individual", "Method of revealing communicative and organizational abilities" by A.V. Batarshev [1-4].

Based on these methods, three levels of development of the studied phenomenon were identified: high, medium, and low. During the ascertaining experiment, it was revealed that most of the students are aware of the need to master communication skills. This indicator is at an average level. As for their need for a future profession and for working internationally, students are aware of the need for mastery and show a high level of motivation. Students believe that communication skills are an important competence for their profession, as many associate their future with the implementation of major national and international projects. However, only a few realize the need for comprehensive work on themselves in order to develop the competencies needed in professional activities. As a result, we can conclude that the motivational component is at an average level.

After conducting specially organized measures, including trainings, role-playing games in order to develop communication skills, we conducted a control diagnosis and determined that the low level decreased, and the high level became higher. The results of the two stages are shown in table 1.

Table 1. Comparison of the results of communicative skills formation (number of people).

\begin{tabular}{|c|c|c|}
\hline Stages & Ascertaining & Control \\
\hline Low & 50 & 13 \\
\hline Medium & 21 & 27 \\
\hline High & 29 & 60 \\
\hline
\end{tabular}

Rokich's method of Value orientations (Research of value orientations of M. Rokich. / Rokich values questionnaire) [6] provided the research of the direction of the individual and determination of his/her attitude to the world, to other people, to itself, the perception of the world, the key motives of actions, the basis of the " philosophy of life".

M. Rokich distinguishes two classes of values - terminal and instrumental. It defines terminal values as beliefs that some ultimate goal of individual existence (for example, a happy family life, world peace) is worth striving from a personal and social point of view [2]. Instrumental values are the beliefs that a certain course of action (for example, honesty, rationalism) is preferable from a personal and public point of view in all situations. In fact, the separation of terminal and instrumental values produces a fairly traditional distinction between values-goals and values-means. The resulting individual hierarchy of values can be divided into three equal groups:

- preferred values, significant (ranks 1 to 6);

- indifferent, indifferent (7-12);

- rejected, insignificant (13-18 rank of the hierarchy). 


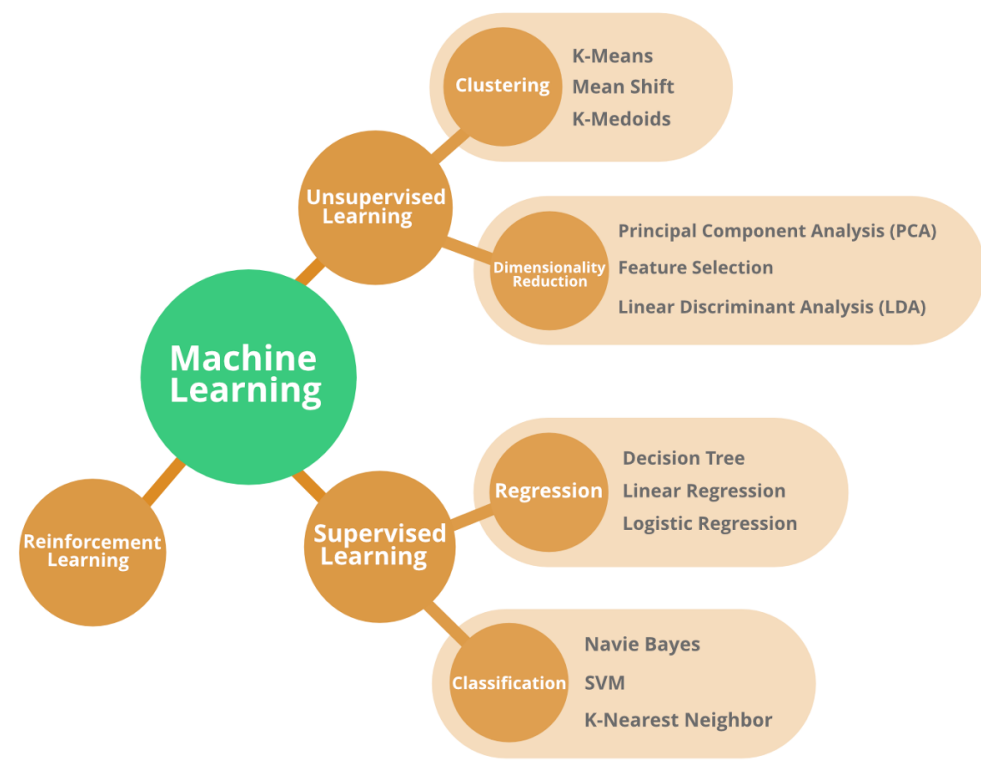

Fig. 1. The average value of grades of terminal and instrumental of Machine learning.

Fig. 1 shows the average values of terminal and instrumental values of future economists after the use of pedagogical technologies. Instrumental values are more developed (responsibility, tolerance, breadth of views (ability to understand someone else's point of view, respect other tastes, customs, habits), honesty).

\section{Conclusion}

The results of our experiment show that professional knowledge and skills, personal moral and person's psychological qualities create the structure of the professional and ethical culture of the manager of the organization. The degree of the communicative qualities development, readiness for communicative interaction plays an important role in hiring. In recent years, professional organizations administration selects such specialists who have the desire for awareness; a high level of claims and ambitious desire to assert their identity; the ability to establish business relationships, the ability to listen and convince.

Clearly established communication channels in an organization allow economists to prevent conflicts, collaborate with the team, and resolve issues as they arise. An economist with good communication skills can relate well with the co-workers and thus, be able to achieve the company's set goals and objectives. The flexible adaptation skills mastering will be particularly useful, since there is a high probability of interpersonal conflicts and a decrease in the labor efficiency while working together, the presence of highly developed group work skills and social communication skills is a necessary condition for hiring economists.

\section{References}

1. T. Wiggins, D. Swift, U. Mai, R. Luechtefeld, 2011 Frontiers in Education Conference (FIE) (2011). doi: 10.1109/fie.2011.6142859 
2. M. Haselkorn, IEEE International Professional Communication Conference, 2003. IPCC 2003. Proceedings (2003). doi: 10.1109/ipcc.2003.1245506

3. R. Reghunath, A. Abdalla, M. Javiad Zaidi, A. Hodges, 2019 IEEE International Professional Communication Conference (Procomm) (2019). doi: 10.1109/procomm.2019.00010

4. M. Dakich, 1995 IEEE International Professional Communication Conference. IPCC 95 Proceedings. Smooth Sailing To The Future (1995). doi: 10.1109/ipcc.1995.554883

5. N. Naik, 2016 IEEE International Symposium On Systems Engineering (ISSE) (2016). doi: 10.1109/syseng.2016.7753150

6. S. Chernyi, V. Budnik, Methods for optimizing solutions when considering group arguments by team of experts. (2017). doi: 10.1063/1.5009873

7. N. Kovalnogova, S. Sokolov, S. Cherny, A. Shnurenko, V. Burlov, 2016 XIX IEEE International Conference On Soft Computing And Measurements (SCM) (2016). doi: $10.1109 / \mathrm{scm} .2016 .7519809$ 